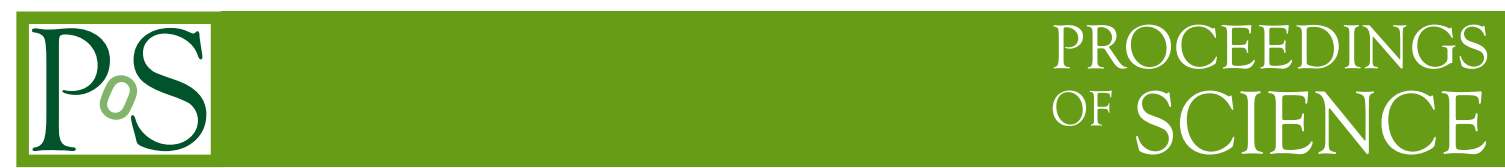

\title{
Dyck words and multi-quark amplitudes
}

\section{Tom Melia*}

CERN Theory Division

E-mail: thomas.melia@cern.ch

In this talk I describe how to use Dyck words and group theory relations to determine the number of independent general QCD primitive amplitudes i.e. involving any number of distinct quarks plus gluons, as described in Ref. [1]. I also present two new tree-level colour decompositions which take a particularly simple form, for the cases of $4 q+n g$ and $6 q+n g$.

11th International Symposium on Radiative Corrections (Applications of Quantum Field Theory to Phenomenology) (RADCOR 2013),

22-27 September 2013

Lumley Castle Hotel, Durham, UK

${ }^{*}$ Speaker. 


\section{Introduction}

Both ATLAS and CMS experiments at the Large Hadron Collider (LHC) observe events with large numbers of hard QCD jets - results with up to ten jets have been published $[2,3]-$ and such final states present a huge theoretical challenge for making reliable predictions in fixed order perturbative QCD, where the next-to-leading order (NLO) corrections are taken into account. At some stage, this fixed order approach is expected to break down in that the $n$th jet in an event will be better described by a parton shower than a hard matrix element, as it will be relatively soft in comparison with the total event (no matter how hard the jets are required to be). Whether this number $n$ is $10,9,8,7, \ldots$ etc. is an interesting question, and there will be large-statistics LHC data at such jet multiplicities to explore this regime.

Although inclusive jet production is dominated by more gluonic sub-processes, there is a lot of interesting multi-quark phenomenology at the LHC. Experimental groups are increasingly applying jet substructure techniques $[4,5]$ in order to discriminate between light quark jets and gluon jets $[6,7,8]$ - these are used, for example in searches for supersymmetric particle cascade decays, where most light jets are light-quark jets. The use of $b$-tagging techniques in many searches directly increases the multi-quark content of the amplitudes needed to calculate cross-sections where they are applied, and electroweak bosons in the final state require quark lines to be present in order to couple to. New physics searches which look in high- $p_{T}$ tails of distributions get a increased contribution from valence quark annihilation, with the result that the more multi-quark amplitudes are numerically important.

In this talk, I will be focusing on properties of general QCD primitive amplitudes, in particular those with multiple quark lines - this additional structure alters the group theory relations which these amplitudes satisfy, with interesting results. These primitive amplitudes can be used in both tree-level and, via unitarity based techniques, in one-loop calculations (see e.g. [9] for a review). They increasingly become a more efficient way of calculating than a Feynman diagram expansion as the number of legs, $n$, becomes very large, as it does for multi-jet processes - the use of recursion relations (such as Berends-Giele recursion [10]) to obtain them directly avoids the quick growth in complexity that accompanies the factorial increase with $n$ of the number of contributing Feynman diagrams.

Understanding the properties of these amplitudes allows for them to be used more efficiently in phenomenological calculations of multi-jet processes. An understanding of a basis for these primitives also allows for the construction of new colour decompositions for the $4 q+n g$ and $6 q+n g$ cases which take a simple form - in particular, they involve the minimal number of primitive amplitudes necessary.

\section{Dyck words and multi-quark amplitudes}

Consider amplitudes for $g^{n-2 k}(\bar{q} q)^{k}$ scattering at tree-level, with $k$ distinct flavours of quark line. A useful way to define primitive amplitudes when there are quarks involved is to consider a theory in which the quarks and antiquarks are charged under the adjoint representation of $S U(3)$. 


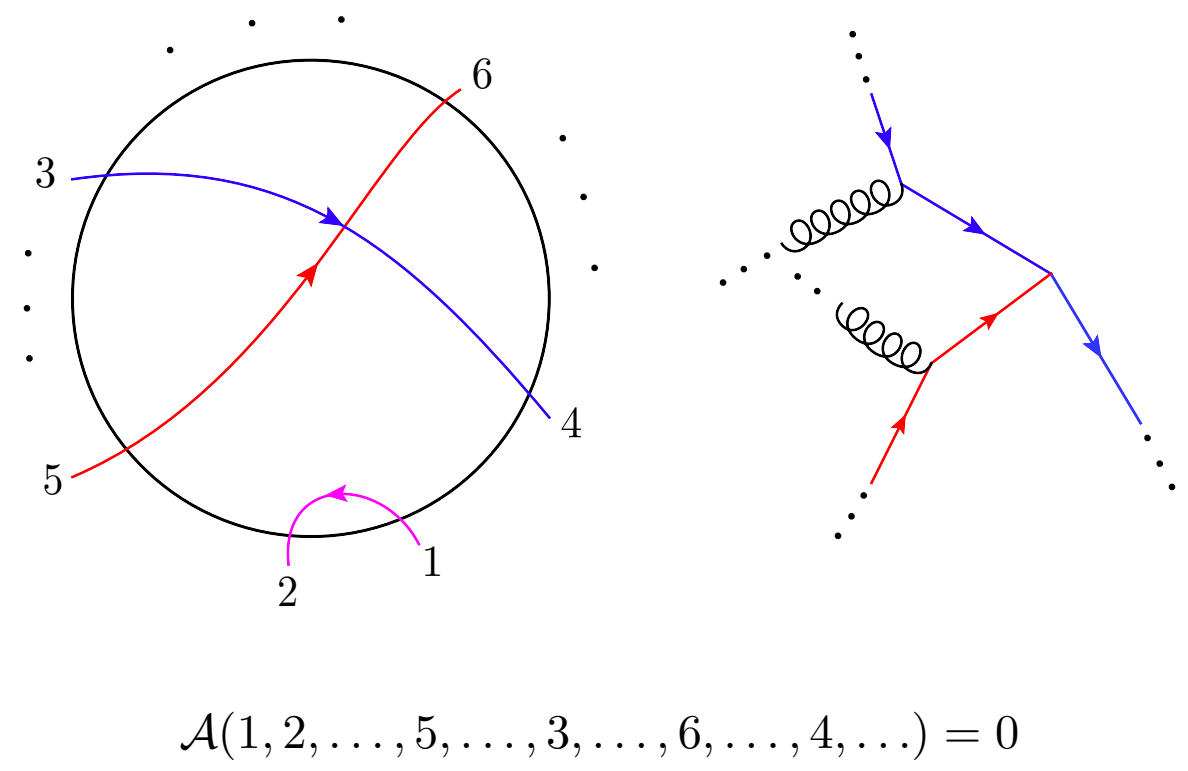

Figure 1: On the lhs is a quark line graph corresponding to the primitive above - it has crossed quark lines. The rhs shows the unavoidable flavour-violating vertex in all contributions to this primitive.

Then the colour decomposition [11]

$$
\mathscr{M}^{\text {tree }}=\sum_{\sigma \in S_{n-1}} \operatorname{tr}\left(\lambda^{1} \lambda^{\sigma_{1}} \ldots \lambda^{\sigma_{n-1}}\right) \mathscr{A}\left(1, \sigma_{1}, \sigma_{2}, \ldots, \sigma_{n-1}\right)
$$

defines the purely kinematic primitive amplitudes $\mathscr{A}$ - however, being purely kinematic, they are just the same as they are in normal QCD. These primitive amplitudes are gauge invariant, and are planar and cyclically ordered. In the pure gluon case, only $(n-2)$ ! of these amplitudes are independent, as they satisfy the Kleiss-Kuijf (KK) relations [12],

$$
\mathscr{A}(1, \alpha, 2, \beta)=(-1)^{n_{\alpha}} \sum_{\sigma \in O P\left(\alpha^{T}, \beta\right)} \mathscr{A}(1,2, \sigma)
$$

where $\alpha^{T}$ is the set alpha reversed, $n_{\alpha}$ is the size of the set $\alpha$, and $O P\left(\alpha^{T}, \beta\right)$ stands for ordered permutations of $\alpha^{T}$ and $\beta$, which is the shuffle product of the two sets. In this way, two gluons can be fixed in the cyclic ordering. Moving away from the pure gluon case, the KK relations still apply, because they are purely group theory relations and follow from making such a colour decomposition eq. 2.1 - as such, two labels can still be fixed. But now there are further relations between the primitives $\mathscr{A}(1,2, \sigma)$ which are induced by the quark line structure and which reduce the number of independent to fewer than $(n-2)$ !. In the first place, some of the primitives with distinct-flavour quark lines are zero. A useful way to see this is to draw a quark line diagram see the lhs of Fig. 1. The quark labels of the primitive (gluon labels are ignored in these diagrams) are written around the edge of a circle representing the edge of the plane on which the primitive 


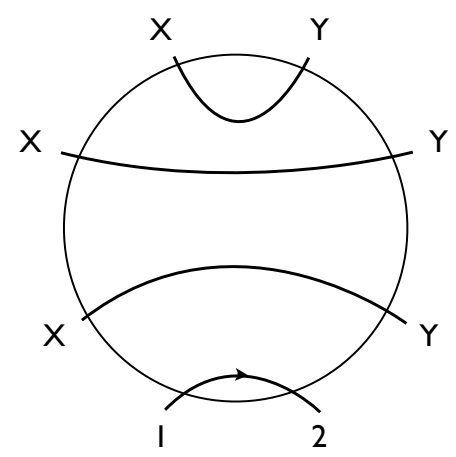

XXXYYY

Figure 2: An example of how to draw a quark line topology for the Dyck word $X X X Y Y Y$.

is defined, and lines are drawn in and out of the circle to connect quarks of equal flavour. In the case shown in Fig. 1, these are the flavour pairs $(\bar{q}, q)$ labelled $(1,2),(3,4)$, and $(5,6)$ (all particles are taken to be outgoing). A useful aspect of these diagrams is that if these quark lines cross, then every contribution to the primitive will have to have a flavour-violating interaction, given that the primitives are planar and cyclically ordered (see rhs of Fig. 1).

A way of counting the non-zero amplitudes of the form $\mathscr{A}(1,2, \sigma)$ is by using Dyck words. Dyck words are strings of $r$ letter $X \mathrm{~s}$ and $r$ letter $Y \mathrm{~s}$ with the property that the number of $X \mathrm{~s}$ is always greater than or equal to the number of $Y \mathrm{~s}$ in any initial segment of the string. For example:

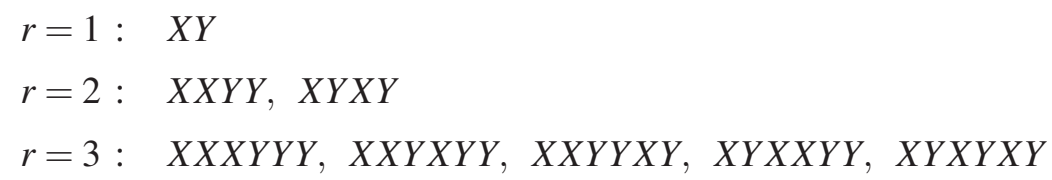

The number of Dyck words of length $2 r$ is given by the Catalan number $C_{r}=(2 r) ! / r !(r+1) !$. They give all possible non-crossing topologies of quark line graph, if the Dyck word is written out around the edge of a the circle, and moving left to right through the Dyck word, each $Y$ is connected with quark line to the most recently encountered, unconnected $X$ (for an example, see Fig. 2). All possible flavour choices of the quark lines must be considered, but only one choice of the direction of the arrow on the line is independent (this follows through a group theory relation, given in Ref. [1]). This gives rise to a counting for the pure quark case (where $r=n / 2-1$ ) as

$$
\begin{aligned}
& \#(\text { Dyck words }) \cdot \#(\text { Flavour allocations }) \\
& =\frac{(2 r) !}{r !(r+1) !} \cdot r !=\frac{(n-2) !}{\left(\frac{n}{2}\right) !}
\end{aligned}
$$

This generalises easily to the case with $g^{n-2 k}(\bar{q} q)^{k}$ with $k$ distinct flavour of quark lines - the number of general QCD primitives is $(n-2) ! / k !$. 


\section{Two new simple colour decompositions}

Colour decompositions have long been known for $n$ gluons [11, 13] and $2 q+n$ gluons [14] for general $n$. I will present new colour decompositions for the cases $4 q+n g$ and $6 q+n g$. The method is based around 'unique Feynman diagrams' (UFDs). The problem can be stated in terms of a Feynman diagram expansion as follows:

$$
\begin{aligned}
& \mathscr{A}_{i}=\sum_{j \in \text { colour ordered }} D_{j} \\
& \mathscr{M}^{\text {tree }}=\sum_{i=1}^{\text {\#basis }} \mathscr{C}_{i}^{\mathrm{Amp}} \mathscr{A}_{i}=\sum_{k=1}^{\# F D} C_{k} D_{k} .
\end{aligned}
$$

The first equation states that a primitive $\mathscr{A}_{i}$ can be expressed as a sum over all contributing purely kinematic parts of the Feynman diagrams, $D_{i}$, calculated using colour ordered Feynman rules [15]. The second equation expresses the full matrix element in two ways - firstly as a sum over a basis of primitives with unknown colour coefficients $\mathscr{C}_{i}$, and secondly as a sum over all Feynman diagrams (i.e. $D_{i}$ multiplied by their usual colour factors from the Feynman rules). This is a system of linear equations which can be solved to find the $\mathscr{C}_{i}$. However, the system becomes trivial to solve if one $D_{x}$ is unique to an amplitude $\mathscr{A}_{x}$ - i.e. it is a UFD - because then it follows from eq. 3.1 that $\mathscr{C}_{x}=C_{x}$. An understanding of the bases of primitive amplitudes allows one to maximise the number of UFDs and this leads to simple expressions for the colour decompositions. Beyond the cases shown, the same techniques render the linear system easily solvable.

\subsection{Case $4 q+n g$}

A useful choice of basis for this case is the set of primitives $\left\{\mathscr{A}\left(1, \mathbf{n}_{1}, 2, \mathbf{n}_{\mathbf{2}}, 3, \mathbf{n}_{\mathbf{3}}, 4\right)\right\}$, where 1 and 2 are antiquark and quark respectively of flavour $f_{1}, 3$ and 4 are antiquark and quark of flavour $f_{2}$, and the $\mathbf{n}_{\mathbf{i}}$ are sets of gluon labels which are a splitting of some permutation of the $n$ gluon labels, that is, $\left\{\mathbf{n}_{\mathbf{1}}, \mathbf{n}_{\mathbf{2}}, \mathbf{n}_{\mathbf{3}}\right\}=\mathbf{n}=\left\{\sigma_{1}, \sigma_{2}, \ldots, \sigma_{n}\right\}$. The basis consists of all possible splittings, and all possible permutations $\sigma \in S_{n}$. Each of these primitive amplitudes has a UFD which looks like

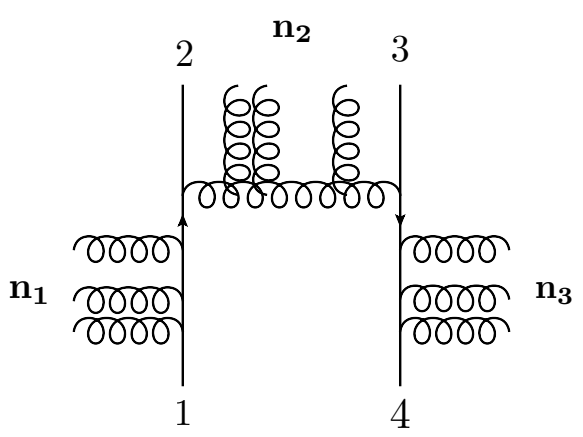

That this is unique follows because flipping any of the legs at any of the vertices of the graph produces a cyclic ordering of external particles which is outside the basis just specified. The colour decomposition then follows by multiplying each of the basis primitives by the colour factor of the 
above diagram, evaluated using the normal Feynman rules:

$$
\left(\lambda^{n_{1}^{1}} \lambda^{n_{1}^{2}} \ldots \lambda^{n_{1}^{\left|\mathbf{n}_{1}\right|}} \lambda^{a}\right)_{2}^{1}\left[F^{n_{2}^{1}} F^{n_{2}^{2}} \ldots F^{n_{2}^{\left|\mathbf{n}_{2}\right|}}\right]_{b}^{a}\left(\lambda^{b} \lambda^{n_{3}^{1}} \lambda^{n_{3}^{2}} \ldots \lambda^{n_{3}^{\left|\mathbf{n}_{3}\right|}}\right)_{4}^{3},
$$

where $n_{i}^{p}$ is the $p$ th element in $\mathbf{n}_{\mathbf{i}},\left|n_{i}\right|$ is the number of elements in the set $\mathbf{n}_{\mathbf{i}},\left[F^{a}\right]_{c}^{b}=i f^{b a c}$, and where the indices 1,2,3,4 appearing are the colour indices of the (anti)quarks, and where there is a summation over the indices $a$ and $b$. We can use the shorthand notation $\left(\lambda \sigma^{\sigma_{1}} \ldots \lambda \sigma^{\sigma_{n}}\right)_{j}{ }_{j} \equiv$ $\left(\sigma_{1} \ldots \sigma_{n}\right)_{j}^{i}$ and $\left[F^{\sigma_{1}} \ldots F^{\sigma_{n}}\right]_{b}^{a} \equiv\left[\sigma_{1} \ldots \sigma_{n}\right]_{b}^{a}$. Because the above diagrams are a UFD to each basis primitives, the colour decomposition follows easily with eq. 3.2 simply multiplying each primitive:

$$
\mathscr{M}(4 q+n g)=\sum_{\mathbf{n} \in S_{n}} \sum_{\left\{\mathbf{n}_{1}, \mathbf{n}_{2}, \mathbf{n}_{3}\right\}=\mathbf{n}}\left(\mathbf{n}_{1} a\right)_{2}^{1}\left[\mathbf{n}_{\mathbf{2}}\right]_{b}^{a}\left(b \mathbf{n}_{3}\right)^{3}{ }_{4} \mathscr{A}\left(1, \mathbf{n}_{\mathbf{1}}, 2, \mathbf{n}_{\mathbf{2}}, 3, \mathbf{n}_{\mathbf{3}}, 4\right),
$$

where the second summation is over all possible splittings.

\subsection{Case $6 q+n g$}

A useful choice of basis in this case is the set of primitives $\left\{\mathscr{A}\left(1, \mathbf{n}_{\mathbf{1}}, 2, \mathbf{n}_{\mathbf{2}}, 3, \mathbf{n}_{\mathbf{3}}, 4, \mathbf{n}_{\mathbf{4}}, 5, \mathbf{n}_{\mathbf{5}}, 6\right)\right\}$ together with the primitives $\left(\left\{\mathscr{A}\left(1, \mathbf{n}_{\mathbf{1}}, 2,3, \mathbf{n}_{\mathbf{2}}, 5, \mathbf{n}_{3}, 6, \mathbf{n}_{\mathbf{4}}, 4, \mathbf{n}_{\mathbf{5}}\right)\right\}+\right.$ cyclic $\left.(1,2),(3,4),(5,6)\right)$, where the antiquark-quark pairs of equal flavour are labelled $(1,2),(3,4),(5,6)$, and now the gluon list $\mathbf{n}$ is split up into five different sets $\mathbf{n}_{\mathbf{1}}$ to $\mathbf{n}_{\mathbf{5}}$. The UFDs for these primitives are
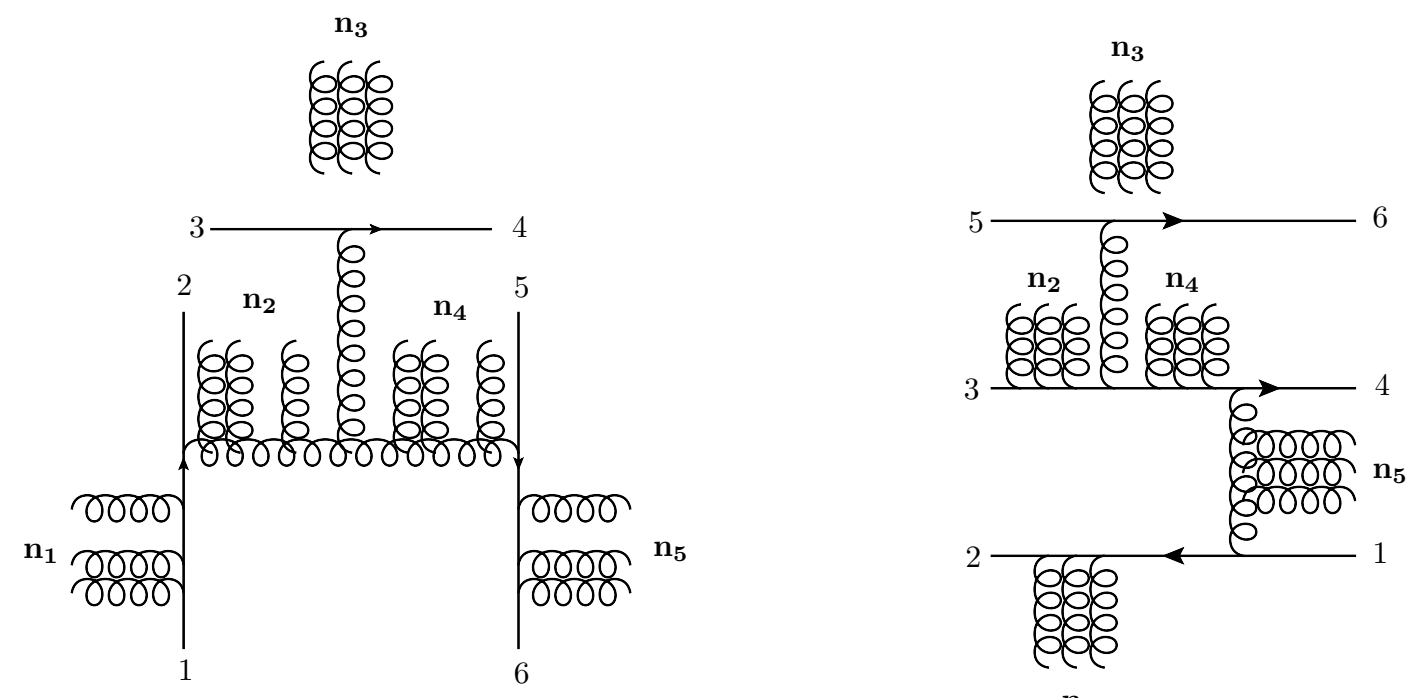

$\mathbf{n}_{1}$

where a difference now lies in that the comb representing the gluon set $\mathbf{n}_{\mathbf{3}}$ is not attached to a quark line in the above pictures - if it is, then flipping the legs on each of the vertices of this comb will now create a different cyclic ordering of external particles which is in our chosen basis. There is a small and trivial system of of linear equations to solve - it is simple because of the comb structure of the gluons in this particular diagram, and the use of such little combs can be extended to higher numbers of quarks, if desired, making inversion easy (although the number of terms would require automation on a computer). The solution of this system modifies the $\mathbf{n}_{\mathbf{3}}$ part of the usual Feynman 
rules associated with the colour factors of the above diagrams. The colour decomposition is

$$
\begin{array}{r}
\mathscr{M}(6 q+n g)=\sum_{\mathbf{n} \in S_{n}} \sum_{\left\{\mathbf{n}_{\mathbf{1}}, \mathbf{n}_{\mathbf{2}}, \mathbf{n}_{\mathbf{3}}, \mathbf{n}_{\mathbf{4}}, \mathbf{n}_{\mathbf{5}}\right\}=\mathbf{n}} \\
\left(\mathbf{n}_{\mathbf{1}} a\right)_{2}{ }_{2}\left[\mathbf{n}_{\mathbf{2}}\right]_{b}^{a} \mathscr{T}_{g}\left(\mathbf{n}_{\mathbf{3}}, 3,4\right)^{b}{ }_{c}\left[\mathbf{n}_{\mathbf{4}}\right]_{d}^{c}\left(d \mathbf{n}_{\mathbf{1}}\right)_{6}^{5}{ }_{6} \mathscr{A}\left(1, \mathbf{n}_{\mathbf{1}}, 2, \mathbf{n}_{\mathbf{2}}, 3, \mathbf{n}_{\mathbf{3}}, 4, \mathbf{n}_{\mathbf{4}}, 5, \mathbf{n}_{\mathbf{5}}, 6\right) \\
+\left[\left(a \mathbf{n}_{\mathbf{1}}\right)_{2}{ }_{2}\left(\mathbf{n}_{\mathbf{2}}\right)_{i}^{3} \mathscr{T}_{q}\left(\mathbf{n}_{\mathbf{3}}, 5,6\right)^{i}{ }_{j}\left(\mathbf{n}_{\mathbf{4}} b\right)^{j}{ }_{4}\left[\mathbf{n}_{\mathbf{5}}\right]_{a}^{b} \mathscr{A}\left(1, \mathbf{n}_{\mathbf{1}}, 2,3, \mathbf{n}_{\mathbf{2}}, 5, \mathbf{n}_{\mathbf{3}}, 6, \mathbf{n}_{\mathbf{4}}, 4, \mathbf{n}_{\mathbf{5}}\right)\right. \\
+\operatorname{cyclic}(1,2)(3,4)(5,6)],
\end{array}
$$

where the modifications to the Feynman rules for the $\mathbf{n}_{\mathbf{3}}$ gluons gives rise to

$$
\begin{aligned}
& \mathscr{T}_{g}(\mathbf{s}, \bar{q}, q)_{b}^{a}=\sum_{\mathbf{s}_{\mathbf{1}}, \mathbf{s}_{\mathbf{2}} \in \text { ord. subs. } \mathbf{s}}\left(a \mathbf{s}_{\mathbf{1}}\right)_{q}^{\bar{q}}\left[a \mathbf{s}_{\mathbf{2}}\right]_{b}^{a}, \\
& \mathscr{T}_{q}(\mathbf{s}, \bar{q}, q)^{i}{ }_{j}=\sum_{\mathbf{s}_{\mathbf{1}}, \mathbf{s}_{\mathbf{2}} \in \text { ord. subs. } \mathbf{s}}\left(a \mathbf{s}_{\mathbf{1}}\right)_{q}^{\bar{q}}\left(a \mathbf{s}_{\mathbf{2}}\right)_{j}^{i},
\end{aligned}
$$

where ' $\mathbf{s}_{\mathbf{1}}, \mathbf{s}_{\mathbf{2}} \in$ ord. subs. $\mathbf{s}$ ' means: $\mathbf{s}_{\mathbf{1}}$ is a subset of the set $\mathbf{s}$ with the ordering of the elements of $\mathbf{s}$ preserved, and $\mathbf{s}_{\mathbf{2}}$ are the remaining elements, also with ordering preserved.

Both of the new colour decompositions are directly in terms of primitive amplitudes, and only rely on the minimum number needed to express the full amplitude.

\section{Conclusion}

In this talk I have shown that tree-level primitive amplitudes in QCD have an interesting mathematical structure, and outlined how to construct a basis around Dyck words for $g^{n-2 k}(\bar{q} q)^{k}$ scattering with $k$ flavours of quark lines. The number of independent primitives is $(n-2) ! / k !$. The understanding of relations between primitives and a minimal set should be useful in the organisation and efficiency of multi-jet LHC phenomenological calculations. I presented two new all- $n$ colour decompositions for $4 q+n g$ and $6 q+n g$, which take a simple form, and are directly given in terms of a minimal set of primitives.

Note added: After this talk was presented, Ref. [16] appeared with an alternative approach to colour decomposition that leads to all- $n$ formulas, the results of which which take a different form to the ones presented above.

\section{References}

[1] Tom Melia. Dyck Words and Multi-Quark Primitive Amplitudes. 2013.

[2] Georges Aad et al. Search for new phenomena in final states with large jet multiplicities and missing transverse momentum at $\sqrt{s}=8 \mathrm{TeV}$ proton-proton collisions using the ATLAS experiment. JHEP, 1310:130, 2013.

[3] CMS Collaboration, CMS-PAS-SUS-13-012. Search for New Physics in the Multijets and Missing Momentum Final State in Proton-Proton Collisions at 8 TeV. 2013. 
[4] Jason Gallicchio and Matthew D. Schwartz. Quark and Gluon Tagging at the LHC. Phys.Rev.Lett., 107:172001, 2011.

[5] Andrew J. Larkoski, Gavin P. Salam, and Jesse Thaler. Energy Correlation Functions for Jet Substructure. JHEP, 1306:108, 2013.

[6] Light-quark and Gluon Jets in ATLAS. 2011.

[7] Serguei Chatrchyan et al. Search for a Higgs boson in the decay channel $H$ to $Z Z\left(^{*}\right)$ to $q$ qbar $\ell^{-} l^{+}$in pp collisions at $\sqrt{s}=7 \mathrm{TeV}$. JHEP, 1204:036, 2012.

[8] CMS Collaboration, CMS-PAS-JME-13-002. Performance of quark/gluon discrimination in $8 \mathrm{TeV}$ pp data. 2013.

[9] R. Keith Ellis, Zoltan Kunszt, Kirill Melnikov, and Giulia Zanderighi. One-loop calculations in quantum field theory: from Feynman diagrams to unitarity cuts. Phys.Rept., 518:141-250, 2012.

[10] Frits A. Berends and W.T. Giele. Recursive Calculations for Processes with n Gluons. Nucl.Phys., B306:759, 1988.

[11] Michelangelo L. Mangano, Stephen J. Parke, and Zhan Xu. Duality and Multi - Gluon Scattering. Nucl.Phys., B298:653, 1988.

[12] Ronald Kleiss and Hans Kuijf. Multi-gluon cross-sections and give jet production at hadron colliders . Nucl.Phys., B312:616, 1989.

[13] Vittorio Del Duca, Lance J. Dixon, and Fabio Maltoni. New color decompositions for gauge amplitudes at tree and loop level. Nucl.Phys., B571:51-70, 2000.

[14] Michelangelo L. Mangano. The Color Structure of Gluon Emission. Nucl.Phys., B309:461, 1988.

[15] Dixon, Lance J. Calculating scattering amplitudes efficiently. hep-ph/9601359, 1996.

[16] Christian Reuschle and Stefan Weinzierl. Decomposition of one-loop QCD amplitudes into primitive amplitudes based on shuffle relations. 2013. 